\title{
The clinical correlates of suicidal ideation in Parkinson's disease
}

\author{
Daniele Belvisi $^{\mathrm{a}, 1}$, Isabella Berardelli ${ }^{\mathrm{b}, 1}$, Gina Ferrazzano ${ }^{\mathrm{a}}$, Matteo Costanzo ${ }^{\mathrm{c}}$, \\ Valentina Corigliano $^{\mathrm{b}}$, Giovanni Fabbrini ${ }^{\mathrm{a}, \mathrm{c}}$, Alfredo Berardelli ${ }^{\mathrm{a}, \mathrm{c}, *}$, Maurizio Pompili $^{\mathrm{b}}$ \\ a IRCSS NEUROMED, Pozzilli, IS, Italy \\ ${ }^{\mathrm{b}}$ Department of Neurosciences, Mental Health and Sensory Organs, Suicide Prevention Center, Sant'Andrea Hospital, Sapienza University of Rome, Italy \\ ${ }^{\mathrm{c}}$ Department of Human Neurosciences, Sapienza University of Rome, Italy
}

\section{A R T I C L E I N F O}

\section{Keywords:}

Parkinson's disease

Suicidal ideation

Perceived disability

Motor symptoms

Non-motor symptoms

\begin{abstract}
A B S T R A C T
Introduction: Suicidal ideation is increased in Parkinson's disease (PD) patients. The possible relationship between parkinsonian motor and non-motor symptoms and suicidal ideation in PD is unknown. The aim of the study was to evaluate suicidal ideation in patients with PD in a controlled study specifically designed to identify the clinical correlates of PD suicidal ideation.

Methods: We consecutively enrolled 100 patients with PD, 76 patients with psoriasis and 80 healthy subjects. Motor symptoms and complications were evaluated by using the International Parkinson and Movement Disorder Society-Unified Parkinson's Disease Rating Scale. Non-motor symptoms were evaluated by means of the Non-Motor Symptoms assessment scale for PD. All patients also underwent a psychiatric evaluation that included the administration of the Columbia-Suicide Severity Rating Scale.

Results: Suicidal ideation was present in $31 \%$ of PD patients, $16 \%$ of patients with psoriasis and $2 \%$ of healthy controls. PD patients showing suicidal ideation have more frequent motor complications, more severe non-motor symptoms and a higher perceived disability than patients without suicidal ideation. Suicidal ideation correlated with the presence of motor complications as well as with non-motor symptom severity, perceived disability severity and the presence of psychiatric disorders.

Conclusions: Our findings showed that suicidal ideation is increased in PD patients compared with healthy controls and patients with psoriasis and that several clinical PD features, including motor complications and nonmotor symptoms, are associated with suicidal ideation in PD. The results suggest a multi-factorial origin of PD suicidal ideation.
\end{abstract}

\section{Introduction}

Several studies have consistently reported that Parkinson's disease (PD) patients have a higher suicidal ideation rate than the general population without performing a comparison with a control group of healthy subjects [1-3]. PD is a chronic, progressive neurological disorder characterized by disabling motor and non-motor symptoms [4]. The possible influence of PD motor features, including motor symptoms, motor complications and motor subtypes, on suicidal ideation is still unclear. One study reported that the risk of suicide is higher among patients in whom motor symptoms onset occurs in the upper limbs or is generalized [1-3]. The authors did not, however, evaluate suicidal ideation in that study. Another study instead excluded the possibility that motor symptoms and motor complications play a major role in suicidal ideation in PD [1]. The relationship between suicidal ideation and motor subtype has never been investigated [5].

PD patients may also display a wide range of non-motor symptoms, such as psychiatric disturbances, pain, sleep disorders and cardiovascular, urinary and gastrointestinal dysfunctions. All these symptoms represent an important source of disability in PD patients [4,6]. Although a relationship between suicide risk and psychiatric disorders in PD has been suggested $[1,2,7]$, to date no studies have investigated the possible influence of other parkinsonian non-motor symptoms on PD suicidal ideation. The extent to which the interaction between motor

Abbreviations: C-SSRS, Columbia Suicide Severity Rating Scale; FAB, Frontal Assessment Battery; H\&Y, Hoehn and Yahr scale; IDPS, Italian Perceived Disability Scale; LEDDs, levodopa-equivalent daily dose; MoCA, Montreal Cognitive Assessment; MDS, UPDRS; Movement Disorder Society-sponsored revision of the Unified Parkinson's Disease Rating Scale, NMSS; Non-Motor Symptoms assessment scale for Parkinson's disease, PD:Parkinson's disease

* Corresponding author. Department of Human Neurosciences, "Sapienza" University of Rome, Viale dell'Università 30, 00185 Rome, Italy.

E-mail address: alfredo.berardelli@uniroma1.it (A. Berardelli).

${ }^{1}$ Dr Daniele Belvisi and Dr Isabella Berardelli have provided an equal contribute to the paper. 
and non-motor symptoms contributes to PD suicidal ideation is thus unknown.

The aim of the present controlled study was to assess the relationship between suicidal ideation and the clinical features of PD, including motor symptoms, motor complications and motor subtype as well as non-motor symptoms. The results were compared with those observed in a control group of healthy subjects. Since it is known that chronic medical conditions are associated to an increased suicidal ideation [8], we also compared our results with those obtained in a control group of patients affected by psoriasis. Our hypothesis was that several clinical factors likely interact and contribute to suicidal ideation in PD.

\section{Methods}

\subsection{Subjects}

One-hundred patients (men: 63\%; mean age: $65.83 \pm 10.25$ ), 80 healthy subjects (men: $62 \%$; mean age: $64.9 \pm 5.9$ years) and 76 patients with psoriasis (men: $52 \%$; mean age: $62.2 \pm 7.4$ ) participated in the study. PD patients were consecutively enrolled from the outpatient department of Human Neurosciences, Sapienza, University of Rome. In our PD group no patients underwent surgical treatment for PD. For the control group of healthy subjects, age- and sex-matched subjects were randomly enrolled from a pool of voluntary subjects with no history of neurological disorders. The diagnosis of PD was based on clinical criteria $[9,10]$. The Montreal Cognitive Assessment (MoCA) [11] and the Frontal Assessment Battery (FAB) [12] were used to evaluate the presence of cognitive impairment. To avoid any bias due to cognitive impairment, all the patients with a MoCA score lower than 24 [13], or a FAB score lower than 12 were excluded from the study [14]. Each patient underwent a neurological and psychiatric evaluation. The study was approved by the local ethics committee of Sapienza, University of Rome and written informed consent was obtained from all the patients prior to enrolment.

\subsection{Neurological evaluation}

We collected the PD patients' demographic and clinical data, including age, gender, marital status, employment, age at onset, disease duration and dopaminergic medication dosages (Table 1). The patients' treatment was evaluated and their levodopa-equivalent daily dose (LEDDs) calculated. No patients were on anti-dyskinetic drugs.The neurological assessment included the evaluation of the disease stage by means of the Hoehn and Yahr scale (H\&Y) [15]. The severity of motor symptoms was assessed by administering the Italian version of the International Parkinson and Movement Disorders Society-sponsored revision of the Unified Parkinson's Disease Rating Scale (MDS-UPDRS) (part III) [16]. Motor complications, including off periods and dyskinesia, were evaluated by means of the MDS-UPDRS part IV. To investigate the relationship between suicidal ideation and motor subtype, we identified motor subtypes by using the Stebbins' method that is based on MDS-UPDRS parts II and III [17]. Following this method we classified PD patients as tremor dominant (TD), postural instability/gait disorder (PIGD) and intermediate subtype. As proposed by Stebbins et al., we calculated the TD/PIGD score ratio for each patient by dividing the tremor score by the PIGD score. Patients with a ratio lower than 0.90 were classified as PIGD while patients with a ratio higher than 1.15 were classified as TD. Patients with a ratio between 0.90 and 1.15 were classified in the intermediate subtype.

Non-motor symptoms were evaluated by means of the Non-Motor Symptoms assessment scale for PD (NMSS) [18]. The NMSS is based on different domains related to cardiovascular symptoms, sleep disturbances, autonomic dysfunction, cognitive impairment, neuropsychiatric dysfunction and pain.

\subsection{Psychiatric evaluation}

PD patients underwent a full psychiatric evaluation performed by two experienced psychiatrists. The psychiatric diagnosis was made on the basis of the Structured Clinical Interview (SCID-5) for DSM-5. In PD patients, healthy controls and patients with psoriasis the severity of depression was assessed by administering the Beck Depression Inventory (BDI).

The Columbia Suicide Severity Rating Scale (C-SSRS), the Italian Perceived Disability Scale (IDPS) and the Beck Hopelessness Scale (BHS) were also administered. The $C$-SSRS is a suicidal ideation rating scale created to evaluate suicidality in children aged 12 years upwards [19]. C-SSRS rates the individual's degree of suicidal ideation on a scale ranging from "wish to be dead" to "active suicidal ideation with specific plan and intent." We defined "past suicidal ideation" as the presence of lifetime suicidal ideation. Conversely, the definition of "recent suicidal ideation" implied that the suicidal ideation was present during the last month. We defined "patients with suicidal ideation" as those patients who reported a story of lifetime or recent suicidal ideation as tested by the $C$-SSRS administration. The severity of suicidal ideation was expressed by means of the $C$-SSRS scores.

The IDPS is a 20-item self-report instrument assessing headacherelated disability on a 5-point Likert scale (completely false to completely true) [20]. The IDPS is based on items that investigate people's beliefs regarding autonomy/disability in different conditions of life. Sample items include "my body is weak and unreliable" and "I will have to worry about my health conditions for the rest of my life".

The psychiatric evaluation, including the administration of the $C$ SSRS, was also performed in the 80 healthy subjects and in the 76 patients with psoriasis enrolled.

\subsection{Co-morbidities evaluation}

In order to measure the burden of co-morbidities we used the Charlson Weighted Index (CWI) [21,22]. CWI is a useful tool to classify co-morbidities and to estimate the risk of death resulting from comorbid diseases. CWI includes a number of different conditions that are rated on the base of the risk of death and are scored from 1 to 6 . The global score included a further point for each decade above 40 years. Higher scores indicate greater comorbidity.

\subsection{Statistical analysis}

We used the SPSS 24.0 toolbox (version 24, IBM, New York, USA) for all the statistical analyses. Descriptive statistical analyses were used to identify the percentage of patients with suicidal ideation and calculate the scores of the psychiatric and neurological scales.

In order to compare suicidal ideation (expressed as the C-SSRS scores) and the presence of psychiatric disturbances in PD patients, healthy subjects and patients with psoriasis we used a Kruskall-Wallis test. The post hoc analysis was performed by applying the MannWhitney U -test.

To investigate possible clinical differences between PD patients with and those without suicidal ideation, we used a Mann-Whitney $U$ test. We considered the following demographic and clinical variables for the statistical analysis: age, gender, employment, age at onset, disease duration, stage of disease (H\&Y score), LEDDs, co-morbidities burden (CWI score), motor symptom severity (MDS-UPDRS part III score), presence of motor complications (MDS-UPDRS part IV score), motor subtype (tremor-dominant-PD vs PIGD), non-motor symptom severity (single domain and total NMSS score), perceived disability (IPDS score) and presence of psychiatric diagnosis.

In order to identify factors that may be associated with suicidal ideation in PD, we used a Spearman's rank correlation coefficient to correlate suicidal ideation (expressed in terms of $C$-SSRS scores) with the demographic and clinical variables. 
Table 1

Demographic and clinical features of PD patients with and without suicidal ideation and healthy controls.

\begin{tabular}{|c|c|c|c|c|c|}
\hline & Patients with suicidal ideation & Patients without suicidal ideation & Patients with psoriasis & Healthy controls & $\mathrm{P}$ \\
\hline Age (years) & $63.5 \pm 11.2$ & $66.5 \pm 9.1$ & $62.2 \pm 7.4$ & $64.9 \pm 5.9$ & n.s. \\
\hline Age at onset (years) & $57.1 \pm 11.1$ & $61.4 \pm 9.1$ & 56.7 & - & n.s. \\
\hline Marital status (\% married) & $79 \%$ & $83 \%$ & $73 \% \%$ & $85 \%$ & n.s. \\
\hline Disease duration & $6.4 \pm 3.8$ & $5.1 \pm 3.7$ & $5.5 \pm 3.2$ & - & n.s. \\
\hline LEDDs & $411.6 \pm 212$ & $415.9 \pm 220$ & - & - & n.s. \\
\hline Patients on Levodopa & $74 \%$ & $70 \%$ & - & - & n.s. \\
\hline Patients on dopamine agonist & $41 \%$ & $40 \%$ & - & - & n.s. \\
\hline Patients on MAO inhibitor & $54 \%$ & $59 \%$ & - & - & n.s. \\
\hline Patients on COMT inhibitor & $0 \%$ & $3 \%$ & - & - & n.s. \\
\hline MDS-UPDRS part III & $24.5 \pm 10.6$ & $20.6 \pm 9.1$ & - & - & n.s. \\
\hline $\mathrm{H} \& \mathrm{Y}$ & $2.1 \pm 0.7$ & $1.8 \pm 0.7$ & - & - & n.s. \\
\hline TD subtype & $39 \%$ & $48 \%$ & - & - & n.s \\
\hline PIGD subtype & $53 \%$ & $41 \%$ & - & - & n.s \\
\hline Intermediate subtype & $8 \%$ & $11 \%$ & - & - & n.s \\
\hline Off periods & $47 \%$ & $24 \%$ & - & - & 0.01 \\
\hline Dyskinesias & $30 \%$ & $16 \%$ & - & - & 0.02 \\
\hline Major depression & $36 \%$ & $7 \%$ & $13 \%$ & $2 \%$ & 0.00004 \\
\hline Persistent Depressive Disorder (Dysthymia) & $12 \%$ & $4 \%$ & $6 \%$ & $1 \%$ & 0.0001 \\
\hline Generalized anxiety disorder & $9 \%$ & $10 \%$ & $8 \%$ & $1 \%$ & 0.00002 \\
\hline Panic disorder & $2 \%$ & $2 \%$ & $1 \%$ & $1 \%$ & n.s. \\
\hline Agoraphobia & $1 \%$ & $0 \%$ & $0 \%$ & $0 \%$ & n.s. \\
\hline Social phobia & $1 \%$ & $1 \%$ & $0 \%$ & $0 \%$ & n.s. \\
\hline Post-traumatic stress disorder/Adjustement disorder & $27 \%$ & $14 \%$ & $0 \%$ & $0 \%$ & 0.00003 \\
\hline Psychosis & $6 \%$ & $2 \%$ & $0 \%$ & $0 \%$ & 0.001 \\
\hline Impulse control disorder & $7 \%$ & $3 \%$ & $0 \%$ & $0 \%$ & 0.003 \\
\hline Subjects on antidepressants (\%) & $31 \%$ & $5 \%$ & $11 \%$ & $1 \%$ & 0.001 \\
\hline Duration of treatment with antidepressants (years) & $3.5 \pm 1.7$ & $3.1 \pm 1.6$ & $3.6 \pm 1.6$ & $2.7 \pm 1.1$ & n.s. \\
\hline Patients on antipsychotics (\%) & $3 \%$ & $1 \%$ & $0 \%$ & $0 \%$ & n.s. \\
\hline
\end{tabular}

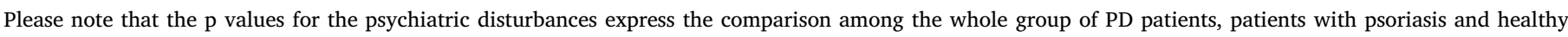
controls.

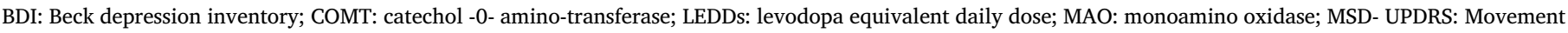
Disorder Society-sponsored revision of the Unified Parkinson's Disease Rating Scale; H\&Y: Hohen and Yahr Scale; PD: Parkinson's disease; n.s: non significant.

To see whether PD suicidal ideation was associated with the demographic and clinical features we also used a logistic regression model with suicidal ideation (present or absent) as a dependent variable.

All the results are reported at $\mathrm{p}<0.05$ after FDR correction for multiple comparisons.

\section{Results}

\subsection{Suicidal ideation: PD patients, healthy subjects and patients with psoriasis}

The clinical and the demographic features of PD patients, healthy controls and patients with psoriasis are reported in Table 1 . The $C$-SSRS scores showed that past suicidal ideation was present in $31 \%$ of the PD patients while recent suicidal ideation was present in 15\%. The 15 patients with recent suicidal ideation also had past suicidal ideation. Past suicidal behaviours were reported by 3 patients (1 preparatory behaviour, 1 aborted attempt, 1 actual non-fatal attempt). In patients with psoriasis the past suicidal ideation was present in $16 \%$ while recent suicidal ideation was present in $9 \%$. In the healthy subjects, past suicidal ideation was present in $2 \%$ while recent suicidal ideation was absent in all. The Kruskall Wallis test showed that the C-SSRS scores significantly differed in the three groups. The post hoc analysis revealed that PD patients had higher $C$-SSRS scores than patients with psoriasis $(\mathrm{p}=0.00003)$ and healthy controls $(\mathrm{p}<0.000001)$. Similarly, the presence of psychiatric disturbances was significantly different in three groups $(p=0.0005)$. The post hoc analysis showed that PD patients showed more frequently psychiatric disturbances than patients with psoriasis ( $p=0.0001)$ and healthy controls $(p<0.00001)$.

IDPS score was significantly higher in PD patients than in patients with psoriasis $(\mathrm{p}=0.005)$. Conversely, CWI scores did not significantly differ in PD patients and patients with psoriasis ( $\mathrm{p}>0.05$ ).

\subsection{Clinical features of suicidal and non-suicidal PD patients}

The Mann-Whitney $U$ test showed that patients with suicidal ideation did not differ from patients without suicidal ideation in terms of age, age at onset, gender, marital status, employment, disease duration or LEDDs, H\&Y and MDS-UPDRS part III scores ( $\mathrm{p}>0.05$ ). The CWI score was similar in patients with $(\mathrm{CWI}: 4.3 \pm 2.1)$ and without suicidal (CWI:3.3 \pm 1.7 ) ideation. Motor complications were more frequent in patients with suicidal ideation than in patients with non-suicidal ideation (off periods: $47 \%$ patients with suicidal ideation, $24 \%$ patients without suicidal ideation; dyskinesias: $30 \%$ patients with suicidal ideation, $16 \%$ patients without suicidal ideation) (Table 1). The motor subtypes (TD/PIGD/intermediate) of PD patients with and without SI are reported in Table 1.

Psychiatric disturbances were present in $85 \%$ patients with suicidal ideation and in $46 \%$ patients without suicidal ideation. Psychiatric disturbances were significantly more frequent in PD patients than in patients with psoriasis and healthy controls (Table 1). Among the various psychiatric disturbances major depression was significantly more frequent in PD patients with suicidal ideation that in PD patients without suicidal ideation $(\mathrm{p}=0.0001)$. PD patients with suicidal ideation showed higher BDI scores than PD patients without suicidal ideation ( $\mathrm{p}<0.0001$ ). In our population of PD patients only 4 patients had a family history of depression (2 patients with suicidal ideation and 2 patients without suicidal ideation). Nineteen percent of patients with suicidal ideation and $3 \%$ of patients without suicidal ideation had a history of depression preceding the diagnosis of PD. No patients had a history of psychosis before the diagnosis of PD. In a further analysis, we used a Mann Whitney $U$ test to compare PD patients with and without ideation, by excluding those patients who had a psychiatric condition preceding the diagnosis of PD. We confirmed that depression was more frequent in patients with suicidal ideation than in patients without suicidal ideation $(\mathrm{p}=0.003)$ and that patients with suicidal ideation 
Table 2

Non motor symptoms severity in PD patients with and without suicidal ideation.

\begin{tabular}{llll}
\hline NMSS domain & $\begin{array}{l}\text { Patients with } \\
\text { suicidal ideation }\end{array}$ & $\begin{array}{l}\text { Patients without } \\
\text { Suicidal ideation }\end{array}$ & $\mathrm{p}$ \\
\hline $\begin{array}{l}\text { 1. Cardiovascular including } \\
\quad \text { falls }\end{array}$ & $1.7 \pm 2.9$ & $1.3 \pm 2.2$ & $\mathrm{n} . \mathrm{s}$ \\
2. Sleep/fatigue & $9.3 \pm 9.9$ & $6.2 \pm 5.2$ & 0.005 \\
3. Mood/cognition & $11.6 \pm 11$ & $5.3 \pm 5.5$ & 0.001 \\
4. Perceptual/hallucinations & $2.1 \pm 3.8$ & $0.7 \pm 2$ & 0.002 \\
5. Attention/memory & $2 \pm 3.2$ & $2 \pm 3.1$ & n.s \\
6. Gastrointestinal tract & $5.2 \pm 5.1$ & $4.2 \pm 4.1$ & n.s. \\
7. Urinary & $6.1 \pm 6.7$ & $4.4 \pm 4.1$ & n.s. \\
8. Sexual function & $3.7 \pm 5.2$ & $2.1 \pm 4.4$ & 0.01 \\
9. Pain & $1.1 \pm 0.9$ & $0.8 \pm 0.7$ & n.s. \\
10. Smell/taste & $2.1 \pm 1.8$ & $2.2 \pm 0.9$ & n.s. \\
Total NMSS & $44.8 \pm 50$ & $29.2 \pm 32.2$ & 0.01 \\
\hline
\end{tabular}

Each item evaluates the frequency and severity separately, and a score ranging from 0 to 12 is obtained by multiplying both concepts. NMSS: non motor symptoms scale; n.s: non significant.

had higher BDI scores than patients without $(\mathrm{p}=0.002)$. By excluding subjects with depression we observed that suicidal ideation was present in $17 \%$ of PD patients, $10 \%$ of patients with psoriasis and none of healthy controls.

By comparing the 15 patients with recent suicidal ideation with those who had past suicidal ideation and with healthy controls, we found that psychiatric disturbances were more frequent in patients with recent suicidal ideation than in healthy controls $(\mathrm{p}<0.01)$ but there were no differences in psychiatric disturbances between patients with past suicidal ideation and patients with recent suicidal ideation (p > 0.05).

Patients with suicidal ideation had significantly higher IPDS ( $p<0.00003$ ) and NMSS scores than patients without suicidal ideation (Table 2). Among the various domains included in the NMSS (Table 2), we observed that patients with suicidal ideation displayed higher rates of sleep disorders, sexual dysfunction and mood/cognition disturbances than patients without suicidal ideation. Although PD patients showed higher BHS scores than healthy controls ( $p<0.001$ ), we did not find any significant difference in BHS score between PD patients with and without suicidal ideation $(\mathrm{p}>0.05)$.

\subsection{Relationship between suicidal ideation and PD clinical features}

Spearman's rank correlation coefficient showed that suicidal ideation (expressed in terms of $C$-SSRS scores) significantly correlated with motor complications $(\mathrm{r}=0.22 ; \mathrm{p}<0.02)$, psychiatric disturbances $(\mathrm{r}=0.47, \mathrm{p}=0.0000008)$, IPDS scores $(\mathrm{r}=0.37 ; \mathrm{p}=0.0001)$ and NMSS scores $(r=0.25 ; \mathrm{p}<0.001)$. Among the single NMSS domains, we found a significant correlation between suicidal ideation and scores in the $\operatorname{mood} / \operatorname{cognition}(\mathrm{r}=0.34 ; \mathrm{p}=0.0005)$, perceptual problem/ hallucination $(\mathrm{r}=0.21 ; \mathrm{p}<0.001)$, sleep disorders $(\mathrm{r}=0.27$; $\mathrm{p}<0.005)$ and sexual dysfunction $(\mathrm{r}=0.24 ; \mathrm{p}=0.001)$ domains. Perceived disability (expressed as the IPDS scores) significantly correlated with the H\&Y score $(r=0.39 ; \mathrm{p}=0.00005)$, MDS-UPDRS score $(\mathrm{r}=0.31 ; \mathrm{p}<0.001)$, presence of motor complications $(\mathrm{r}=0.31$; $\mathrm{p}=0.001)$, the NMSS score $(\mathrm{r}=0.48 ; \mathrm{p}<0.0000004)$ and the presence of psychiatric disorders $(r=0.39 ; p=0.00005)$. Among the various psychiatric disturbances observed in our population, we found a positive correlation between suicidal ideation in PD and major depression $(\mathrm{r}=0.41 ; \mathrm{p}=0.00001)$. Accordingly, BDI scores positively correlated with suicidal ideation $(r=0.39 ; p=0.0001)$.

The logistic regression model showed the suicidal ideation was significantly associated with perceived disability (expressed as IPDS scores) (beta:0.45, $\mathrm{p}=0.02$ ) and the presence of psychiatric disturbances (beta:0.82; $\mathrm{p}=0.003$ ).

\section{Discussion}

Our findings showed that suicidal ideation is increased in PD patients when compared with healthy subjects and patients with psoriasis. A further finding of our study is that PD patients that exhibit suicidal ideation have more frequent motor complications, more severe nonmotor symptoms and a higher perceived disability than patients without suicidal ideation. Suicidal ideation correlated with the presence of motor complications as well as with non-motor symptom severity, perceived disability severity and the presence of psychiatric disorders.

In the present study we observed that suicidal ideation is present in a high percentage of patients affected by PD. Two previous studies showed a similar occurrence of suicidal ideation in PD [1,7], while another study showed a significantly lower value [2]. Previous studies did not include healthy controls and only performed a comparison between the data seen in PD patients with those observed in the general population. Conversely, we compared suicidal ideation in PD patients with that of an age- and sex-matched population of healthy controls enrolled in the same study and with patients affected by another chronic disease, i.e. psoriasis. Several studies have reported that a number of medical chronic conditions are associated with an increased suicidal ideation and with high rates of suicide, suggesting that a medical chronic condition per se could be a risk factor for suicide [8]. The interaction between suicidal ideation and medical chronic conditions is complex [23,24]. The increase of suicidal ideation may depend on the psychological impact of a diagnosis that implies a lifetime physical condition or, alternatively, the pathophysiology underlying various medical illnesses may contribute to increase suicidal ideation [8]. In our study, we observed that PD and psoriasis, had both an increased suicidal ideation when compared with healthy controls. By directly comparing PD and patients with psoriasis, we observed that suicidal ideation was more frequent and more severe in PD than in patients with psoriasis. This means that suicidal ideation is increased in PD patients not simply due to the fact that PD is a chronic disease and that the intrinsic clinical features of PD may render these patients more susceptible to suicidal ideation. In PD patients we studied, the severity of motor symptoms and the stage of the disease were not correlated with suicidal ideation in PD. This is in line with previous evidence showing that motor symptoms in PD are not associated with either suicidal ideation or suicide risk [1,3]. It should be, however, considered that our population was characterized by a mild to moderate PD and therefore we cannot fully exclude that suicidal ideation might increase in the advanced stages of the disease. We also investigated the relationship between PD suicidal ideation and motor subtypes. Similarly to motor symptoms, motor subtype (TD/PIGD/intermediate) did not seem to influence or to be associated with suicidal ideation in PD. By contrast, we observed that motor complications, including off periods and dyskinesia, were more frequent in suicidal PD patients and found a significant correlation between suicidal ideation and motor complications. This is in contrast to a previous study by Nazem et al. [1] who ruled out any association between motor complications and suicidal ideation in PD. This discrepancy between our study and their study might, however, be due to methodological differences as we used a clinical scale that was specifically designed to evaluate suicidal ideation and is highly sensitive to suicidal thoughts [19], whereas Nazem et al. used a modified version of the Paykel scale [25], which included only two items on death ideation and two items on suicidal ideation [1]. Previous studies [26] suggested that motor complications, particularly dyskinesia are not associated with poor quality of life but in the present study we found a significant correlation between the presence of motor complications and perceived disability. This correlation suggests that motor complications may contribute to PD suicidal ideation by increasing the perceived disability, which correlated with higher suicidal ideation in our PD patients.

Interestingly, we observed that a large number of non-motor 
symptoms, including, sleep disorders, hallucinations, perceptual problems and sexual dysfunction, were both more severe and more frequent in PD patients with suicidal ideation than in those without, and significantly correlated with suicidal ideation which means such symptoms may be predictors of suicidality. The association between PD non-motor symptoms and suicidal ideation in PD may depend on a number of factors. Not only do non-motor symptoms make a marked contribution to PD patients' disability [6], but the results of the present study also revealed a strong correlation between non-motor symptom severity and perceived disability. Non-motor symptoms might therefore contribute to suicidal ideation by interacting with perceived disability. It should also be borne in mind, however, that some of the non-motor symptoms that correlated with suicidal ideation in our study, such as hallucinations and sleep disorders, are known to be characteristic of the malignant subtype of PD identified in previous studies [27]. It is, therefore, possible that higher suicidal ideation is a specific feature of the PD malignant subtype.

In line with previous studies [1,2,7], we observed that psychiatric disturbances were related to suicidal ideation in PD. Major depression was significantly more frequent in PD patients with suicidal ideation than in patients without suicidal ideation and the presence of suicidal ideation was positively correlated with the major depression. It is known that depression is an important risk factor for suicidal ideation [28] and Brown et al. [29] have suggested that PD patients with depression had relatively common suicidal ideation, but reduced frequency of attempted and completed suicide. Accordingly, in our study we observed that the frequency of suicidal ideation was high and was also associated with depression and that suicidal behaviours were present in a low percentage of patients.

Our results suggest that the increased perceived disability of parkinsonian patients may play a key role in PD suicidal ideation. Perceived disability is defined as the patient's beliefs regarding the illness, is closely linked to self-efficacy and may affect individual adaptation to a medical disease [30]. In the present study, we also observed that perceived disability in PD is higher in patients with suicidal ideation than in patients without suicidal ideation. The increased perceived disability was significantly associated with suicidal ideation and correlated with the motor and non-motor variables considered.

Overall, our findings suggest that suicidal ideation in PD is related to several clinical features of PD. Some of these features, such as motor complications and non-motor symptoms, exert a direct influence on suicidal ideation. Others, such as motor symptoms, do not appear to be directly related to suicidal ideation but may exert an effect that is mediated by the increased perceived disability, which correlates with both motor and non-motor symptoms. According to this hypothesis, the increased perceived disability that characterizes PD patients may represent a pivotal crossroads between motor and non-motor features that determines suicidal ideation in PD. In summary, our assessment of a large number of demographic and clinical features revealed a complex scenario that points to a multi-factorial origin of suicidal ideation in PD, which is in keeping with current theories on suicidal ideation [31].

The presence co-morbidites is considered a risk factor for suicide in the general population [32]. In our study, however, we found that comorbidities burden was similar in patients with and without suicidal ideation. This finding is consistent with a recent study performed by $\mathrm{Li}$ et al. [20]. showing that co-morbidities are not increased in PD patients with suicidal ideation.

The most obvious limitation of our study is its cross-sectional design. This implies, for instance, that we were not able to evaluate the effect of changes of pharmacological treatment on several clinical factors that are correlated with suicidal ideation in PD, such as off periods and dyskinesia. Thus, the relationships between variables that emerge from this study should be interpreted with caution as follow-up investigations are needed to confirm these findings. A further limitation of our study was that we did not consider some social factors possibly influencing suicidal ideation, including the caregiver support and the financial burden of the disease. Similarly, a limitation of our study was that we did not explore some frontal functions, like insight and decision making that may be implicated in suicidal ideation. In addition, we did not investigate apathy by using specific clinical scales. Finally, we administered some self-report measures, potentially biased by social desirability.

In conclusion, our study has provided the first evidence of the relationship between motor and non-motor clinical features and suicidal ideation in PD. We observed that suicidal ideation is increased in PD patients and that non-motor symptoms, motor complications and the increased perceived disability are associated to suicidal ideation in PD. We suggest that the clinical assessment of PD patients should include a psychiatric evaluation investigating suicidal ideation and perceived disability.

\section{Funding}

Nothing to declare.

\section{Conflicts of interest}

Nothing to declare.

\section{Authors' role}

1. Research project: A. Conception, B. Organization, C. Execution; 2. Statistical Analysis: A. Design, B. Execution, C. Review and Critique; 3. Manuscript Preparation: A. Writing of the first draft, B. Review and Critique.

D. Belvisi: 1 A,B,C; 2 A,B,C, 3A

I. Berardelli: 1 A,B,C; 2 A,B,C, 3A

G. Ferrazzano: 1C.

M. Costanzo: 1C.

V. Corigliano: $1 \mathrm{C}$.

G. Fabbrini: 3B

A. Berardelli: 1A, 1B, 2A, 3B

A.Pompili: 1A, 1B, 2A, 3B

\section{Acknowledgements}

We thank Dr Lewis Baker for language editing.

\section{References}

[1] S. Nazem, A.D. Siderowf, J.E. Duda, G.K. Brown, T. Ten Have, M.B. Stern, D. Weintraub, Suicidal and death ideation in Parkinson's disease, Mov. Disord. 23 (2008) 1573-1579.

[2] A. Kummer, F. Cardoso, A.L. Teixeira, Suicidal ideation in Parkinson's disease, CNS Spectr. 14 (2009) 431-436.

[3] T. Lee, H.B. Lee, M.H. Ahn, J. Kim, M.S. Kim, S.J. Chung, J.P. Hong, Increased suicide risk and clinical correlates of suicide among patients with Parkinson's disease, Parkinsonism Relat. Disord. 32 (2016) 102-107.

[4] A.H.V. Schapira, K.R. Chaudhuri, P. Jenner, Non-motor features of Parkinson disease, Nat. Rev. Neurosci. 18 (2017) 435-450.

[5] R.C. Helmich, M. Hallett, G. Deuschl, I. Toni, B.R. Bloem, Cerebral causes and consequences of parkinsonian resting tremor: a tale of two circuits? Brain 135 (2012) 3206-3226.

[6] K.R. Chaudhuri, D.G. Healy, A.H. Schapira, National Institute for clinical excellence, non-motor symptoms of Parkinson's disease: diagnosis and management, Lancet Neurol. 5 (2006) 235-245.

[7] V.S. Kostić, T. Pekmezović, A. Tomić, M. Jecmenica-Lukić, T. Stojković, V. Spica, M. Svetel, E. Stefanova, I. Petrović, E. Dzoljić, Suicide and suicidal ideation in Parkinson's disease, J. Neurol. Sci. 289 (2010) 40-43.

[8] M. Pompili, A. Forte, A.L. Berman, D.A. Lamis, The association between physical illness/medical conditions and suicide risk, The International Handbook of Suicide Prevention, Wiley \& Sons, Ltd, Chichester, 2016, pp. 133-148.

[9] A. Berardelli, G.K. Wenning, A. Antonini, D. Berg, B.R. Bloem, V. Bonifati, D. Brooks, D.J. Burn, C. Colosimo, A. Fanciulli, J. Ferreira, T. Gasser, F. Grandas, P. Kanovsky, V. Kostic, J. Kulisevsky, W. Oertel, W. Poewe, J.P. Reese, M. Relja, E. Ruzicka, A. Schrag, K. Seppi, P. Taba, M. Vidailhet, EFNS/MDS-ES/ENS recommendations for the diagnosis of Parkinson's disease, Eur. J. Neurol. 20 (2013) $16-34$. 
[10] R.B. Postuma, D. Berg, M. Stern, W. Poewe, C.W. Olanow, W. Oertel, J. Obeso, K. Marek, I. Litvan, A.E. Lang, G. Halliday, C.G. Goetz, T. Gasser, B. Dubois, P. Chan, B.R. Bloem, C.H. Adler, G. Deuschl, MDS clinical diagnostic criteria for Parkinson's disease, Mov. Disord. 30 (2015) 1591-1601.

[11] M. Skorvanek, J.G. Goldman, M. Jahanshahi, C. Marras, I. Rektorova, B. Schmand, E. van Duijn, C.G. Goetz, D. Weintraub, G.T. Stebbins, P. Martinez-Martin, Members of the MDS Rating Scales Review Committee, Global scales for cognitive screening in Parkinson's disease: Critique and recommendations, Mov. Disord. 33 (2018) 208-218.

[12] O.S. Cohen, E. Vakil, D. Tanne, N. Molshatzki, Z. Nitsan, S. Hassin-Baer, The frontal assessment battery as a tool for evaluation of frontal lobe dysfunction in patients with Parkinson disease, J. Geriatr. Psychiatry Neurol. 25 (2012) 71-77.

[13] S. Hoops, S. Nazem, A.D. Siderowf, J.E. Duda, S.X. Xie Stern, M.B. Weint, D. Weintraub, Validity of the MoCA and MMSE in the detection of MCI and dementia in Parkinson, Neurology 73 (2009) 1738-1745.

[14] B. Kaszás, N. Kovács, I. Balás, J. Kállai, Z Aschermann, Z. Kerekes, S. Komoly, F. Nagy, J. Janszky, T. Lucza, K. Karádi, Sensitivity and specificity of addenbrooke's cognitive examination, mattis dementia rating scale, frontal assessment battery and mini mental state examination for diagnosing dementia in Parkinson's disease, Parkinsonism Relat. Disord. 18 (2012) 553-556.

[15] M.M. Hoehn, M.D. Yahr, Parkinsonism: onset, progression and mortality, Neurology 17 (1967) 427-442.

[16] A. Antonini, G. Abbruzzese, L. Ferini-Strambi, B, Tilley, J. Huang, G. T. Stebbins, C.G. Goetz, P. Barone, MDS-UPDRS Italian validation study group, validation of the Italian version of the movement disorder society - unified Parkinson's disease rating scale, Neurol. Sci. 34 (2013) 683-687.

[17] G.T. Stebbins, C.G. Goetz, D.J. Burn, J. Jankovic, T.K. Khoo, B.C. Tilley, How to identify tremor dominant and postural instability/gait difficulty groups with the movement disorder society unified Parkinson's disease rating scale: comparison with the unified Parkinson's disease rating scale, Mov. Disord. 28 (2013) 668-670.

[18] K.R. Chaudhuri, P. Martinez-Martin, R.G. Brown, K. Sethi, F. Stocchi, P. Odin, W. Ondo, K. Abe, G. Macphee, D. Macmahon, P. Barone, M. Rabey, A. Forbes, K. Breen, S. Tluk, Y. Naidu, W. Olanow, A.J. Williams, S. Thomas, D. Rye, Y. Tsuboi, A. Hand, A.H. Schapira, The metric properties of a novel non-motor symptoms scale for Parkinson's disease: results from an international pilot study, Mov. Disord. 15 (2007) 1901-1911.

[19] K. Posner, G.K. Brown, B. Stanley, D.A. Brent, K.V. Yershova, M.A. Oquendo, G.W. Currier, G.A. Melvin, L. Greenhill, S. Shen, J.J. Mann, The Columbia-Suicide
Severity Rating Scale: initial validity and internal consistency findings from three multisite studies with adolescents and adults, Am. J. Psychiatry 168 (2011) 1266-1277.

[20] M. Innamorati, M. Pompili, S. De Filippis, F. Gentili, D. Erbuto, D. Lester, A. Tamburello, G. Iacorossi, I. Cuomo, G. Dominici, R. Tatarelli, P. Martelletti, The validation of the Italian Perceived Disability Scale (IPDS) in chronic daily headache sufferers, J. Headache Pain 10 (2009) 21-26.

[21] W. Li, M.M. Abbas, S. Acharyya, H.L. Ng, K.Y. Tay, W.L. Au, L.C. Tan, Suicide in Parkinson's disease, Mov. Disord. Clin. Pract. 5 (2018) 177-182.

[22] M.E. Charlson, P. Pompei, K.L. Ales, C.R. MacKenzie, A new method of classifying prognostic comorbidity in longitudinal studies: development and validation, J. Chronic Dis. 40 (1987) 373-383.

[23] A.L. Berman, M.M. Silverman, B.M. Bongar, Comprehensive Textbook of Suicidology, Guilford Press, New York, 2000.

[24] T.B. Mackenzie, M.K. Popkin, Medical illness and suicide, in: S.J. Blumenthal, D.J. Kupfer (Eds.), Suicide over the Life Cycle: Risk Factors, Assessment, and Treatment of Suicidal Patients, 1991, pp. 205-232 Washington.

[25] E.S. Paykel, J.K. Myers, J.J. Lindenthal, J. Tanner, Suicidal feelings in the general population: a prevalence study, Br. J. Psychiatry 124 (1974) 460-469.

[26] A.J. Espay, A.E. Lang, Common myths in the use of levodopa in Parkinson disease. When clinical trials misinform clinical practice, JAMA Neurol 74 (2017) 633-634.

[27] S.M. Fereshtehnejad, Y. Zeighami, A. Dagher, R.B. Postuma, Clinical criteria for subtyping Parkinson's disease: biomarkers and longitudinal progression, Brain 140 (2017) 1959-1976.

[28] Y. Conwell, P.R. Duberstein, E.D. Caine, Risk factors for suicide in later life, Soc. Biol. Psychiatry 52 (2002) 193-204.

[29] R.G. Brown, B. MacCarthy, A.M.G.J. Der Gotham, C.D. Marsden, Depression and disability in Parkinson's disease: a follow-up of 132 cases, Psychol. Med. 18 (1988) 49-55.

[30] M. Innamorati, M. Pompili, M. Fiorillo, N. Lala, A. Negro, S.D. Del Bono, D. Lester, P. Girardi, P. Martelletti, Overattachment and perceived disability in chronic migraineurs, Clin. Neurol. Neurosurg. 115 (2013) 954-958.

[31] M. Pompili, M. Belvederi Murri, S. Patti, M. Innamorati, D. Lester, P. Girardi, M. Amore, The communication of suicidal intentions: a meta-analysis, Psychol. Med. 46 (2016) 2239-2253.

[32] D.N. Juurlink, N. Herrmann, J.P. Szalai, A. Kopp, D.A. Redelmeier, Medical illness and the risk of suicide in the elderly, Arch. Intern. Med. 164 (2004) 1179-1184. 